\title{
Jefferson
}

Thomas Jefferson University

HOME OF SIDNEY KIMMEL MEDICAL COLLEGE

The Medicine Forum

Volume 21

Article 3

2020

\section{An Approach to Refractory Hypoxemia}

Daniel Garrido, MD

Thomas Jefferson University, daniel.garrido@jefferson.edu

Evan Nardone, MD

Thomas Jefferson Univeristy, evan.nardone@jefferson.edu

Mark Mallozzi, MD

Thomas Jefferson University, mark.mallozzi@jefferson.edu

Follow this and additional works at: https://jdc.jefferson.edu/tmf

Part of the Internal Medicine Commons

Let us know how access to this document benefits you

\section{Recommended Citation}

Garrido, MD, Daniel; Nardone, MD, Evan; and Mallozzi, MD, Mark (2020) "An Approach to Refractory Hypoxemia," The Medicine Forum: Vol. 21, Article 3.

DOI: https://doi.org/10.29046/TMF.021.1.005

Available at: https://jdc.jefferson.edu/tmf/vol21/iss1/3

This Article is brought to you for free and open access by the Jefferson Digital Commons. The Jefferson Digital Commons is a service of Thomas Jefferson University's Center for Teaching and Learning (CTL). The Commons is a showcase for Jefferson books and journals, peer-reviewed scholarly publications, unique historical collections from the University archives, and teaching tools. The Jefferson Digital Commons allows researchers and interested readers anywhere in the world to learn about and keep up to date with Jefferson scholarship. This article has been accepted for inclusion in The Medicine Forum by an authorized administrator of the Jefferson Digital Commons. For more information, please contact: JeffersonDigitalCommons@jefferson.edu. 


\title{
An Approach to Refractory Hypoxemia
}

\author{
Daniel Garrido, MD, Evan Nardone, MD, Mark Mallozzi, MD
}

\section{INTRODUCTION}

Oxygenation normally occurs through a process of passive diffusion through the pulmonary capillaries, where oxygen binds to hemoglobin or plasma. When the oxygenation is deemed insufficient, a decrease in the partial pressure develops, resulting in hypoxemia. This is in contrast to hypoxia, which develops when there is a low presence of oxygen at the level of the tissue. ${ }^{1.2}$ The oxygenation of hemoglobin is most often measured through the arterial oxygen saturation ( $\mathrm{SaO} 2)$, which represents the proportion of hemoglobin that is bound to oxygen in red blood cells. This is represented most commonly through pulse oximetry, which is normally $>95 \%$ in healthy adults. ${ }^{3}$ To accurately measure the amount of oxygen dissolved in plasma, the arterial oxygen tension ( $\mathrm{PaO} 2$ ) is measured, which is represented through an arterial blood gas (ABG).

One method to measure oxygenation is through the calculation of the A-a oxygen gradient. This measure identifies the difference between oxygenation within the alveoli (PAO2), and within that dissolved in plasma ( $\mathrm{PaO} 2)$. This gradient represents how well gas is exchanged between the alveolar and capillary membranes. When the difference in the A-a gradient ( $\mathrm{PAO} 2-\mathrm{PaO} 2$ ) is normal $(<10 \mathrm{mmHg})$, etiologies for respiratory failure are typically from alveolar hypoventilation or low inspired FiO2. However, when there is a widened A-a gradient, etiologies may include ventilation-perfusion (V/Q) mismatch, right-to-left shunting, or a diffusion defect. ${ }^{4}$ When in the presence of oxygen there is no improvement in a patient's hypoxemia, then the hypoxemia is deemed refractory. Herein we present an unusual case of refractory hypoxemia in a patient with patent foramen ovale (PFO) and extensive deep vein thromboses (DVT).

\section{CASE DESCRIPTION}

A 74-year-old female with no significant pulmonary history presented as a transfer from an outside hospital for persistent refractory hypoxemia. ABG on admission was noted to be pH 7.50, pCO2 25, pO2 58, and HCO3 of 20 while on $13 \mathrm{~L}$ nasal cannula. She was noted to have an A-a gradient $>400$. The patient was also noted to desaturate to $70-80 \%$ O2 saturation with movement and when sitting up (suggestive of orthodeoxia-platypnea syndrome), which continued despite being transitioned to high-flow nasal cannula and intermittent BiPAP therapy. Extensive workup had been performed for her hypoxemia including chest CTA, methemoglobin levels, and autoimmune serologies, which were negative. However, ABG persistently demonstrated $30 \%$ shunt fraction. Transthoracic echocardiogram (TTE) with bubble study was concerning for a PFO. Subsequent transesophageal echocardiogram (TEE) confirmed an ascending aortic aneurysm forcing the PFO open with large and rapid right-to-left shunting. After presuming a right-to-left shunt as the primary cause of hypoxemia, a right heart catheterization (RHC) was performed with an attempted PFO closure (as the patient was not a surgical candidate). RHC demonstrated pulmonary arterial (PA) pressures of $25 \mathrm{mmHg} / 10 \mathrm{mmHg}$. During the procedure a left atrial clot was incidentally found and cleared with catheter removal. However, intra-procedural closure of the PFO resulted in hypotension and no improvement in oxygenation. Given the left atrial clot and the discovery of bilateral lower extremity DVT on ultrasound, a lung perfusion scan was performed to evaluate for chronic thromboembolic disease (as the patient could not tolerate a ventilation scan), however the result was normal. It was ultimately believed that her poor oxygenation was due to her PFO with frequent changes in the shunt due to position and hemodynamics. After a goals of care discussion, the patient was transitioned to comfort care and discharged on hospice.

\section{DISCUSSION}

We believe this case provides a good opportunity to work through a differential for refractory hypoxemia. Despite extensive testing, the ultimate etiology of the presented patient's hypoxemia was unclear at the time of her discharge to hospice. Below we discuss the typical differential for hypoxemia and apply it to the case above.

1. Right-to-left shunt: During right-to-left shunting, hypoxemia is caused by deoxygenated blood mixing with oxygenated blood in the systemic circulation. This can be from anatomic defects such as intracardiac shunts (PFO, VSD, etc.), pulmonary AVMs, or processes causing intrapulmonary vasodilation (i.e. hepatopulmonary syndrome). Shunting can also be physiologic during which non-ventilated lung are perfused which can occur in pneumonia, ARDS, atelectasis, etc. Typically, these patients have hypoxemia, which is difficult to correct with supplemental oxygen. Our patient had an appropriate workup for intracardiac shunting and ultimately a PFO was found on TEE. Furthermore, it was thought 
The Medicine Forum, Vol. 21 [2020], Art. 3

that her ascending aortic aneurysm was forcing her PFO open in certain positions causing her to have positional desaturations. Interestingly, however, when her PFO was occluded during cardiac catheterization, her oxygenation did not improve and ultimately closure was aborted due to hypotension. It is unclear why her oxygenation did not improve with occlusion of her shunt.

2. $\quad V-Q$ mismatch: Some degree of perfusion and ventilation mismatch occurs physiologically in the healthy lung but is worsened in the diseased lung state. Hypoxemia can occur by this mechanism when there is a decrease in the ventilation or perfusion of a lung segment impairing oxygenation of blood. In our patient, given her extensive clot burden with left atrial clot and bilateral lower extremity DVTs, it was thought that pulmonary emboli may have been causing defects in perfusion to her lung and contributing to her hypoxemia. She was unfortunately unable to tolerate a ventilation scan but had a perfusion scan that was normal, making this etiology less likely.

3. Diffusion limitation: Hypoxemia by this mechanism occurs when there is a diminished ability for oxygen to diffuse from the alveolus to the capillary. It is commonly due to interstitial lung disease causing interstitial inflammation and fibrosis. Our patient did not have findings on her chest imaging that were indicative of interstitial inflammation. Additionally, she had an extensive autoimmune serologic workup that was negative. Hypoxemia by this mechanism is commonly worsened by exertion and improved with rest. Our patient had profound hypoxemia at rest making this etiology less likely.

4. Hypoventilation: During hypoventilation, both arterial and alveolar $\mathrm{CO} 2$ increases which causes decrease in alveolar oxygen tension. Hypoxemia due solely to hypoventilation will have a normal A-a gradient, will have primarily elevated $\mathrm{PaCO} 2$, and will rapidly correct with increased $\mathrm{FiO} 2$. A typical presentation may be a patient with obesity hypoventilation, disorders causing respiratory muscle weakness, or CNS depression. Our patient did not have evidence of hypoventilation on her blood gas making this etiology for hypoxemia less likely.

5. Decreased fraction of inspired oxygen (FiO2): This causes hypoxemia by decreasing the oxygen gradient from the alveolus to the artery. This is typically associated with high altitudes and not a likely etiology for our patient given her history.
Ultimately, it was decided that the most likely etiology of our patient's hypoxemia was due to right-to-left intracardiac shunting, but it remains unclear why her hypoxemia did not improve with PFO occlusion during cardiac catheterization. Given her substantial lower extremity clot burden and the presence of intracardiac blood clots, it is possible that V/Q mismatch was also contributing to this patient's hypoxemia with intrapulmonary shunting. However, her right-sided and pulmonary pressures on TTE and RHC did not show evidence of pulmonary hypertension that would be expected in $V / Q$ mismatch due to chronic thromboembolic disease. Although less likely, we cannot rule out this as a contributing factor to her hypoxemia since she was not able to tolerate a full $V / Q$ scan. This case highlights an atypical presentation with multiple possible etiologies of hypoxemia and provides an opportunity to work through a broad differential for this problem.

\section{REFERENCES}

1. Sarkar, M., Niranjan, N., \& Banyal, P. K. (2017). Mechanisms of hypoxemia. Lung India, 34(1), 47-60.

2. Gossman, W., Alghoula, F., \& Berim, I. (2020). Anoxia (Hypoxic Hypoxia). StatPearls. StatPearls Publishing

3. O'Driscoll, B. R., Howard, L. S., Earis, J., Mak, V., British Thoracic Society Emergency Oxygen Guideline Group, \& BTS Emergency Oxygen Guideline Development Group (2017). BTS guideline for oxygen use in adults in healthcare and emergency settings. Thorax, 72(Suppl 1), ii1-ii90.

4. Rudy, C. C., Ballard, C., Broberg, C., \& Hunter, A. J. (2017). PlatypneaOrthodeoxia Syndrome: A Case of Chronic Paroxysmal Hypoxemia. Journal of general internal medicine, 32(1), 127-130. 